\title{
Mapping and modelling helminth infections in ruminants in Europe: experience from GLOWORM
}

Laura Rinaldi ${ }^{1}$, Guy Hendrickx ${ }^{2}$, Giuseppe Cringoli ${ }^{1}$, Annibale Biggeri ${ }^{3}$, Els Ducheyne ${ }^{2}$, Dolores Catelan ${ }^{3}$, Eric Morgan ${ }^{4}$, Diana Williams ${ }^{5}$, Johannes Charlier ${ }^{6}$, Georg von Samson-Himmelstjerna ${ }^{7}$, Jozef Vercruysse ${ }^{6}$

${ }^{1}$ Department of Veterinary Medicine and Animal Productions, University of Naples Federico II, CREMOPAR Campania Region, Naples, Italy; ${ }^{2}$ Avia-GIS, Zoersel, Belgium; ${ }^{3}$ Department of Statistics, Informatics and Applications "G. Parenti", University of Florence, Florence, Italy; ${ }^{4}$ School of Biological Sciences, University of Bristol, Bristol, UK; ${ }^{5}$ Department of Infection Biology/School of Veterinary Science, Institute of Infection and Global Health, University of Liverpool, Liverpool, UK; ${ }^{6}$ Department of Virology, Parasitology and Immunology, Faculty of Veterinary Medicine, Ghent University, Belgium; ${ }^{7}$ Institute for Parasitology and Tropical Veterinary Medicine, Freie Universität Berlin, Berlin, Germany

\begin{abstract}
Mapping and modelling helminth infections in cattle and sheep in Europe through advanced geospatial research was one of the main task of GLOWORM, a three year project (2012-2014) funded under the European Commission's (EC) seventh framework programme (FP7). Liver flukes as Fasciola hepatica and gastrointestinal nematodes, such as Haemonchus contortus were chosen for the project since these parasites constitute a major cause of lost productivity in small and large ruminants. The output of the GLOWORM project delivered guidelines for standardized and harmonized cross-sectional surveys of helminth parasites in ruminants allowing the development of updated prevalence maps and multi-scale, spatial models for the European area.
\end{abstract}

Keywords: helminths, Fasciola hepatica, Haemonchus contortus, mapping, modelling, ruminants, Europe, GLOWORM.

Helminth infections continue to represent a serious challenge to the health, welfare, productivity and reproduction of grazing ruminants throughout the world. In principle, all grazing animals are such exposed to these infections, in particular to liver flukes as Fasciola hepatica and gastrointestinal nematodes (GIN), such as Haemonchus contortus. Together, these parasites constitute a major cause of lost productivity in small and large ruminants as is evident by DISCONTOOLS (http://www.discontools.eu), a programme recently established by the European Union (EU) to prioritize research on animal diseases and identify control tools. The EU is increasingly interested in finding ways and means to limit the impact of these parasites upon animal health, welfare and productivity (Morgan et al., 2013; Charlier et al., 2014; Rinaldi and Cringoli, 2014).

Corresponding author:

Laura Rinaldi

Unit of Parasitology and Parasitic Diseases

Department of Veterinary Medicine and Animal Productions

University of Naples Federico II

Via della Veterinaria 1, 80137 Naples, Italy

$\mathrm{Tel} / \mathrm{Fax}+39081253-6283$

E-mail: lrinaldi@unina.it
The idea behind the current special issue of Geospatial Health is to broaden interest in geospatial research (mapping and modelling) pertaining to helminth infections in large and small ruminants in Europe.

F. hepatica and GIN (in particular H. contortus) were studied in the GLOWORM project: "Innovative and sustainable strategies to mitigate the impact of global change on helminth infections in ruminants", a research consortium running from 2012 to 2014 supported by funds (Grant \# 288975) from the European seventh framework programme (FP7). Ten academic institutions and four small and medium-sized enterprises (SME), comprising a multitude of disciplines, such as parasitology, epidemiology, molecular biology, diagnostics, farming systems, remote sensing and geographical information systems (GIS), from various European countries participated in the project.

The output of the project provided practical and scientific approaches to respond to environmental, climatological and parasitological changes occurring in Europe with special emphasis on:

(i) the design of cost-efficient and practical spatial sampling strategies;

(ii) the construction of a spatial database containing 
environmental and climatic variables for mapping helminth infections; and

(iii) the development of multi-scale, spatio-temporal models for parasite occurrence with special reference to climate change.

Sampling for fasciolosis in cattle based on bulk tank milk (BTM) samples and antibody detection by the enzyme-linked immunosorbent assay (ELISA) has proved itself a well-standardized approach to risk mapping (Ducheyne et al., 2015). Naturally, the presence of specific antibodies reflects exposure to the parasite rather than current infection. However, the main reasons for these kinds of nation-wide surveys are less for finding which farms are infected but rather to document the distribution of an infection. In this case, we were interested in establishing risk maps for stakeholders that range from the farmers and local veterinarians to the various political levels, in particular the decision-makers. Importantly, these risk-maps should be regarded as baseline information that needs to be regularly updated in surveillance programmes. GLOWORM enabled us to produce region- or country-specific such maps (Novobilsky et al., 2015; Selemetas et al., 2015a,b) as well as the first panEuropean map of F. hepatica infection risk in dairy cattle (Ducheyne et al., 2015), a milestone for further work that will be need in the future.

In parallel with the sampling for fasciolosis in dairy cattle, we collected updated and reliable parasitological data for sheep (Rinaldi et al., 2015a,b) through a standardized and harmonized approach based on cost-effi- cient, spatial sampling and diagnostic procedures involving pooled faecal samples and FLOTAC (Cringoli et al., 2010), a highly sensitive and accurate, quantitative and qualitative faecal egg assay.

Parasitological, cross-sectional surveys were conducted using a representative number of cattle and sheep farms in selected pilot areas throughout Europe. The geo-referenced results were used for the creation of maps comprising updated parasitological information on the distribution of F. hepatica and H. contortus infections in ruminant farms in Europe (Bosco et al., 2015; Ducheyne et al., 2015; Rinaldi et al., $2015 \mathrm{a}, \mathrm{b})$. This information was followed by the construction of multi-scale GIS of parasitological, climatic, environmental and farm management data. The data were further subjected to a comparative analysis of parasite occurrence between the study areas distributed throughout Europe using various spatial models, either based on Bayesian geostatistics or advanced machine-learning approaches, such as Random Forests (RF) and Boosted Regression Trees (BRT) with and without environmental and climatic covariates (Ducheyne et al., 2015; Novobilsky et al., 2015; Rinaldi et al., 2015a,b; Selemetas et al., 2015a,b).

Furthermore, the climate-based Ollerenshaw Mt model for F. hepatica was expanded (Caminade et al., 2015) and a $\mathrm{Q}_{0}$ model for $H$. contortus developed, tested and validated (Bolajoko et al., 2015), with a view to the apply these model frameworks on a spatial basis in future.

The output of the GLOWORM project delivered

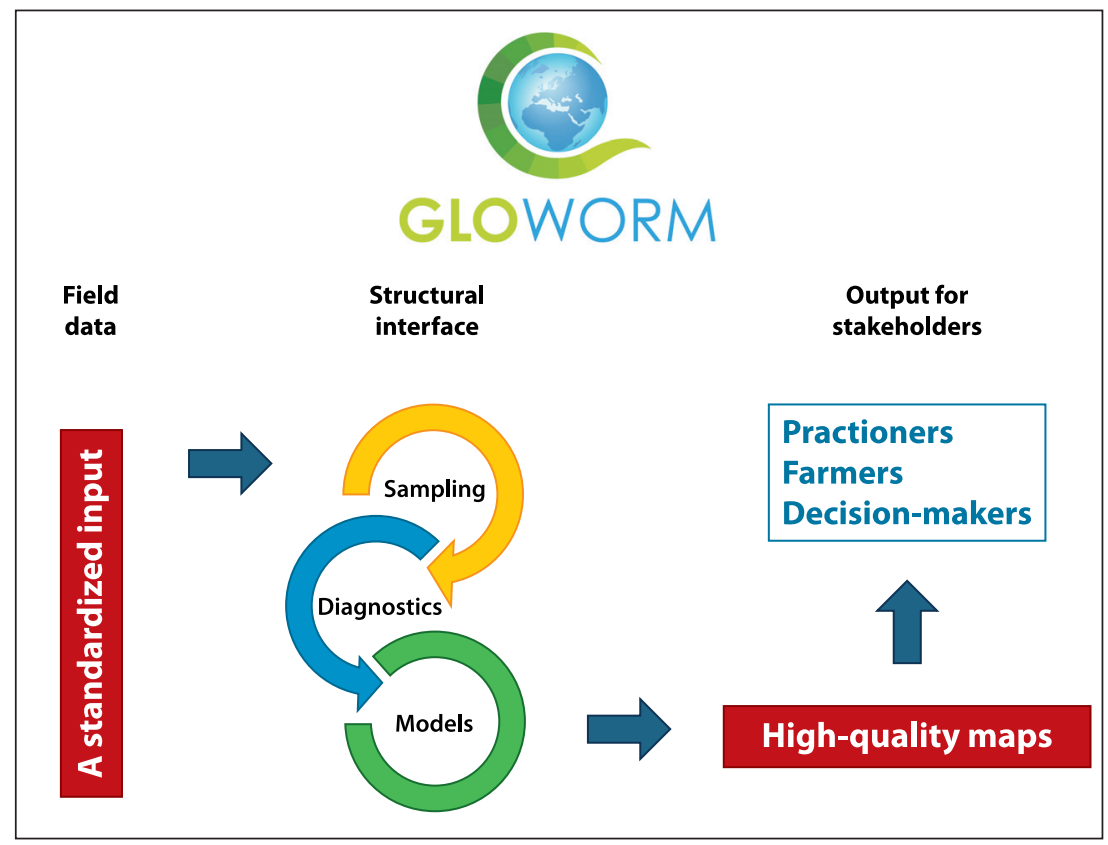

Fig. 1. The GLOWORM interface between sampling, diagnostics and maps. 
guidelines for standardized and harmonized cross-sectional surveys of helminth parasites in ruminants (Ducheyne et al., 2015; Rinaldi et al., 2015a,b) allowing the development of updated prevalence maps and multiscale spatial models for the European area (Fig. 1).

The continued functioning of the ecosystem is vital for the future of farms in Europe, and the findings within the GLOWORM framework reflect the ongoing ecological changes we are witnessing at various levels. To investigate ongoing developments with respect to parasites of veterinary importance, we used a range of models and scenarios of climate and land-use change to conduct this Europe-wide assessment. Although some trends are positive, such as increased agricultural productivity (better growing degree days) or offer opportunities for extension of arable land, many changes increase vulnerability as a result of changes in water availability and soil fertility (Schröter et al., 2005).

The current maps and the climate impact studies demonstrate that both F. hepatica (Caminade et al., 2015) and H. contortus (Bolajoko et al., 2015) already now present an increased risk for health, welfare and productivity of ruminants throughout Europe, a situation that has the potential of becoming worse, even if the climate change of the last 30 years does not undergo further exacerbation as projected by the International Panel on Climate Change (IPCC) (http://www.ipcc.ch/). There are reasons to believe that the spatial occurrence of these parasites, including their transmission periods, will continue to expand in Europe (and other parts of the world). Such a scenario would require increased frequency of prophylactic treatment to control helminth infection, which may well lead to increasing levels of resistance to anthelmintic drugs. Improved control programmes tailored at various multi-scale levels will become a fundamental issue to be seriously considered by animal health authorities. The approaches developed within the GLOWORM project provide important steps towards the application of spatial tools in support of such control programmes.

\section{References}

Bolajoko MB, Rose H, Musella V, Bosco A, Rinaldi L, Van Dijk J, Cringoli G, Morgan ER, 2015. The basic reproduction quotient $\left(\mathrm{Q}_{0}\right)$ as a spatial predictor of the seasonality of ovine haemonchosis. Geospat Health 9, 333-350.

Bosco A, Rinaldi L, Musella V, Amadesi A, Cringoli G, 2015. Outbreak of acute fasciolosis in sheep farms in a Mediterranean area arising as a possible consequence of climate change. Geospat Health 9, 319-324.
Caminade C, Van Dijk J, Baylis M, Williams D, 2015. Modelling recent and future climatic suitability for fasciolosis transmission risk in Europe. Geospat Health 9, 301-308.

Charlier J, Vercruysse J, Morgan E, van Dijk J, Williams DJ, 2014. Recent advances in the diagnosis, impact on production and prediction of Fasciola hepatica in cattle. Parasitology 141, 326-335.

Cringoli G, Rinaldi L, Maurelli MP, Utzinger J, 2010. FLOTAC: new multivalent techniques for qualitative and quantitative copromicroscopic diagnosis of parasites in animals and humans. Nat Protoc 5, 503-515.

Ducheyne E, Charlier J, Vercruysse J, Rinaldi L, Biggeri A, Demeler J, Brandt C, de Waal T, Selemetas N, Höglund J et al., 2015. Modeling the spatial distribution of Fasciola hepatica in dairy cattle in Europe. Geospat Health 9, 261-270.

Morgan ER, Charlier J, Hendrickx G, Biggeri A, Catelan D, von Samson-Himmelstjerna G, Demeler J, Müller E, van Dijk J, Kenyon F et al., 2013. Global change and helminth infections in grazing ruminants in Europe: impacts, trends and sustainable solutions. Agriculture 3, 484-502.

Novobilský A, Sollenberg S, Höglund J, 2015. Distribution of Fasciola hepatica in Swedish dairy cattle and associations with pasture management factors. Geospat Health 9, 293-300.

Rinaldi L, Biggeri A, Musella V, de Waal T, Hertzberg H, Mavrot F, Torgerson P, Selemetas N, Coll T, Bosco A et al., 2015a. Sheep and Fasciola hepatica in Europe: the experience from GLOWORM. Geospat Health 9, 309-317.

Rinaldi L, Catelan D, Musella V, Cecconi L, Hertzberg H, Torgerson P, Mavrot F, de Waal T, Selemetas N, Coll T et al., 2015b. Haemonchus contortus: spatial distribution of risk of infection in sheep in Europe. Geospat Health 9, 325-331.

Rinaldi L, Cringoli G, 2014. Exploring the interface between diagnostics and maps of neglected parasitic diseases. Parasitology 28, 1-8.

Rinaldi L, Levecke B, Bosco A, Ianniello D, Pepe P, Charlier J, Cringoli G, Vercruysse J, 2014. Comparison of individual and pooled faecal samples in sheep for the assessment of gastrointestinal strongyle infection intensity and anthelmintic drug efficacy using McMaster and Mini-FLOTAC. Vet Parasitol 205, 216-223.

Schröter D, Cramer W, Leemans R, Prentice IC, Araújo MB, Arnell NW, Bondeau A, Bugmann H, Carter TR, Gracia CA et al., 2005. Ecosystem service supply and vulnerability to global change in Europe. Science 310, 1333-1337.

Selemetas N, Ducheyne E, Phelan P, O'Kiely P, Hendrickx G, de Waal T, 2015a. Spatial analysis and risk mapping of Fasciola hepatica infection in dairy herds in Ireland. Geospat Health 9, 281-291.

Selemetas N, Phelan P, O'Kiely P, de Waal T, 2015b. Cluster analysis of fasciolosis in dairy cow herds in Munster province of Ireland and detection of major climatic and environmental predictors of the exposure risk. Geospat Health 9, 271-279. 